\title{
Telemonitoramento e resolução espontânea no atraso da erupção dos incisivos centrais superiores durante a pandemia - Relato de caso
}

Telemonitoring and spontaneous resolution in delayed eruption of central incisors during tge pandemic - Case report

Telemonitorización y resolución espontânea em erupción tardía de incisivos centrales durante la pandemia - Reporte de caso

\section{Resumo}

Apesar da erupção dentária ser considerado um processo fisiológico, os Odontopediatras são frequentemente questionados sobre possíveis alterações decorrentes da irrupção. O objetivo deste trabalho é apresentar um caso de atraso na erupção dos incisivos centrais superiores em uma criança do sexo masculino com 8 anos de idade.

Devido a pandemia, e o aconselhamento dos órgãos governamentais suspenderem o atendimento odontológico eletivo, a conduta foi utilizar o telemonitoramento para acompanhar, aconselhar e acolher o paciente. Durante as consultas, de forma remota, foi possível observar que a gengiva do paciente estava fibrosada, uma vez que a criança apresentou perda precoce dos dentes decíduos. Com as imagens fornecidas pela mãe, foi possível observar na região dos dentes 11 e 21 um abaulamento gengival. Após 3 meses de acompanhamento, os dentes irromperam apresentando uma resolução clinica espontânea e de mínima intervenção. Com isso, concluímos um bom diagnóstico realizado pelo cirurgião dentista é essencial para o sucesso na conduta clinica e o uso do telemonitoramento foi uma ferramenta potente e segura neste momento de pandemia permitindo acompanhar à distância as crianças com os cuidados de saúde bucal.

Palavras-chave: Teleodontologia; Odontopediatria; Pandemia.

\begin{abstract}
Although tooth eruption is considered a physiological process, Pediatric Dentists are often questioned about possible changes resulting from irruption. The objective of this paper is to present a case of delayed eruption of maxillary central incisors in an 8-year-old male child. Due to the pandemic, and the advice of government agencies to suspend elective dental care, the conduct was to use telemonitoring to monitor, advise and accommodate the patient. During the consultations, remotely, it was possible to observe that the patient's gums were fibrosed, since the child presented early loss of deciduous teeth. With the images provided by the mother, it was possible to observe in the region of teeth 11 and 21 a gingival bulging. After 3 months of follow-up, the teeth erupted, presenting a spontaneous clinical resolution, with minimal intervention. Thus, we conclude that a good diagnosis made by the dental surgeon is essential for successful clinical management and the use of telemonitoring was a powerful and safe tool at this time of pandemic, allowing remote monitoring of children with oral health care.
\end{abstract}

Keywords: Teleodontics; Pediatric Dentistry; Pandemic. 


\begin{abstract}
Resumen
Aunque la erupción de los dientes se considera un proceso fisiológico, los Dentistas Pediátricos se preguntan a menudo sobre los posibles cambios resultantes de la irrupción. El objetivo de este trabajo es presentar un caso de retraso en la erupción de los incisivos centrales maxilares en un niño de 8 años.

Debido a la pandemia, y al consejo de los organismos gubernamentales de suspender la atención odontológica electiva, la conducta fue utilizar la telemonitorización para controlar, asesorar y acoger al paciente. Durante las consultas, a distancia, se pudo observar que las encías del paciente estaban fibrosadas, ya que el niño presentaba una pérdida temprana de los dientes deciduos. Con las imágenes proporcionadas por la madre, se pudo observar en la región de los dientes 11 y 21 un abultamiento gingival. Tras 3 meses de seguimiento, los dientes erupcionaron con una resolución clínica espontánea y una intervención mínima. Por lo tanto, concluimos que un buen diagnóstico realizado por el cirujano dentista es esencial para el éxito del manejo clínico y el uso de la telemonitorización fue una herramienta poderosa y segura en este momento de la pandemia, permitiendo el seguimiento a distancia de los niños con la atención de la salud oral.
\end{abstract}

Palabras clave: Teleodontología; Odontopediatía; Pandemia.

\title{
1. Introdução
}

A erupção dentária é um dos fenômenos que se manifesta como parte do crescimento e desenvolvimento do organismo, podendo, assim, sofrer a influência das variações que ocorrem no ritmo e na velocidade do crescimento (Rezende, Zollner, \& Santos, 2010). O crescimento somático, por sua vez, tem determinação genética embora seja também bastante influenciado pelos fatores ambientais (socioeconômicos, nutricionais e de urbanização). Fatores nutricionais, além de serem importante no desenvolvimento dos tecidos dentários pode também influenciar no tempo de irrupção dos dentes. Crianças nascidas prematuras e, consequentemente de baixo peso, tendem a ter um atraso no processo de erupção dentária, por outro lado crianças obesas apresentam um tempo de erupção mais precoce. Já crianças que estão no padrão de crescimento e desenvolvimento adequado pela organização mundial da saúde (O.M.S) o período da erupção dentária pode ser baseado nas tabelas de cronologia de erupção dentária, sendo estas um importante parâmetro de normalidade e para que se possa fazer o diagnóstico de possíveis alterações do crescimento e desenvolvimento (Couto, Franco, Bussadori, Santos, \& Rezende, 2021; Haddad \& Correa, 2005). O atraso de erupção dos incisivos centrais permanentes pode gerar dificuldade na socialização de crianças e afligir muito os pais sobre os desdobramentos na dentadura mista e longevidade dessa situação, visto que afeta em muito a estética (Suri, Gagari, \& Vastardis, 2004). Quando há fibrose da mucosa gengival, o dente permanente pode perder a força eruptiva não sendo capaz de penetrar no tecido gengival. Diversos são os motivos pelos quais isso pode acontecer: fatores locais devido ao mau posicionamento do germe do dente permanente, mucosa muito espessa, dentes anquilosados, perda prematura ou retenção prolongada, algumas síndromes, fatores hereditários ou até mesmo desordens endócrinas. O tratamento é acompanhamento e ponderar se há necessidade cirúrgica ou não (Dodson \& Susarla, 2014; Schneider et al., 2014).

A nova doença coronavírus 2019 (COVID-19) chamou a atenção depois que o primeiro caso foi relatado em dezembro de 2019 em Wuhan China e desde então, afetou quase todos os países do mundo e foi declarada uma pandemia pela OMS (Credie, Coelho, \& Rezende, 2020; Meng, Hua, \& Bian, 2020; Paules, Marston, \& Fauci, 2020). Na área da odontologia, a pandemia de COVID-19 interrompeu o atendimento clinico, seja de consultório privado, seja da educação odontológica globalmente, já que a maioria das escolas e universidades de odontologia do mundo foram fechadas como forma de medida preventiva ("lockdown"), e distanciamento social, prejudicando o acompanhamento e tratamento dos pacientes. Frente a isso, utilizar a teleconsulta/telediagnóstico/telemonitoramento no intuito de respaldar os pacientes nesse período foi uma alternativa (Freitas, Carvalho, Alves, Costa, \& Carvalho, 2021). A ciência tem nos permitido usar a comunicação eletrônica para dividir informações, buscar conselhos especializados e opiniões através das distâncias (Jarvis \& Stanberry, 2005). A teleconsulta/telediagnóstico/telemonitoramento encurtou essas distancias, permitindo a comunicação entre profissionais oferecendo suporte adequado para garantir a resolubilidade e a qualidade do atendimento à população em locais isolados, com poucos recursos e nesse período de pandemia (Golder \& Brennan, 2000; Revista de Odontopediatría Latinoamericana, 2021). 
Assim, o objetivo deste trabalho é relatar o caso clinico de uma criança de 8 anos de idade, no qual estava com queixa e baixa auto estima devido ao atraso da erupção dos dentes 11 e 21 durante a pandemia e foi adotado o acompanhamento e telemonitoramento até período de erupção dentária.

\section{Metodologia}

O presente trabalho é um relato de um caso clinico sobre o atraso de erupção dentária dos incisivos centrais superiores permanentes de uma criança que não conseguia atendimento odontológico devido a pandemia COVID-19. A alternativa encontrada foi utilizar o telemonitoramento como forma de assistência. Antes de iniciar cada consulta, o responsável recebia um formulário criado pela plataforma google por email com o termo de consentimento livre e esclarecido, autorizando a utilização de dados clínicos, imagens fotográficas que se encontram em sua ficha de prontuário odontológico para finalidades científicas. Portanto, levando em consideraçã os princípios éticos que regem a pesquisa com seres humanos presentes na Declaração de Helsinque.Somente após a aceitação, a realização da ligação era iniciada.

\section{Relato de Caso}

No mês de agosto de 2020, a responsável de uma criança de 8 anos, sexo masculino, gostaria de agendar uma consulta odontológica para seu filho com a queixa da ausência dos dentes 11 e 21 .

Durante a anamnese virtual, a mãe relatou que seu filho sofreu um trauma dental quando ele tinha 2 anos de idade e os incisivos centrais decíduos superiores avulsionaram mas não chegou a procurar nenhum atendimento odontológico naquele momento. Quando a criança estava com 6 anos de idade (2019) ela resolveu marcar uma consulta com um cirurgião dentista porque seu filho não apresentava nenhum sinal clinico de erupção dos incisivos centrais permanentes superiores. Na ocasião, o dentista realizou uma radiografia panorâmica (foto 1), avaliou o estágio de formação radicular dos dentes permanentes e a tranquilizou informando que a qualquer momento os dentes iriam irromper. Após 1 ano e 2 meses da tomada da radiografia panorâmica, a mãe continuou preocupada uma vez que os dentes não haviam irrompidos e que a criança estava se sentindo diferente, incomodada com a situação além de estar atrapalhando na fala e na alimentação porque a gengiva estava inchada e sensível. Como forma de orientação para aliviar a sensibilidade e dor na gengiva foi indicado compressas de gelo e alimentos gelados como sorvetes, água gelada além de reforçar a higiene bucal com pasta fluoreatada acima de 1000 ppmF.

O telemonitoramento foi realizado uma vez por semana e após cada conversa a mãe encaminhava uma foto da arcada bucal do seu filho como forma de registro (figura 2). Acompanhando as imagens, após 3 meses do primeiro contato, observamos que no dia 27 de novembro havia a presença das incisais dos dentes 11 e 21 . No dia 20 de dezembro as incisais dos dentes $11 \mathrm{e}$ 21 já estavam expostas e em 13 de janeiro de 2021 dois terços da coroa presentes na cavidade bucal.

Assim, o paciente com quadro clinico a gengiva fibrosada devido à perda prematura dos incisivos decíduos, apresentou uma resolução fisiológica do processo de erupção dos dentes não necessitando de intervenção cirúrgica. 
Research, Society and Development, v. 10, n. 7, e18010716439, 2021

(CC BY 4.0) | ISSN 2525-3409 | DOI: http://dx.doi.org/10.33448/rsd-v10i7.16439

Foto 1: Radiografia panorâmica inicial, junho de 2019.

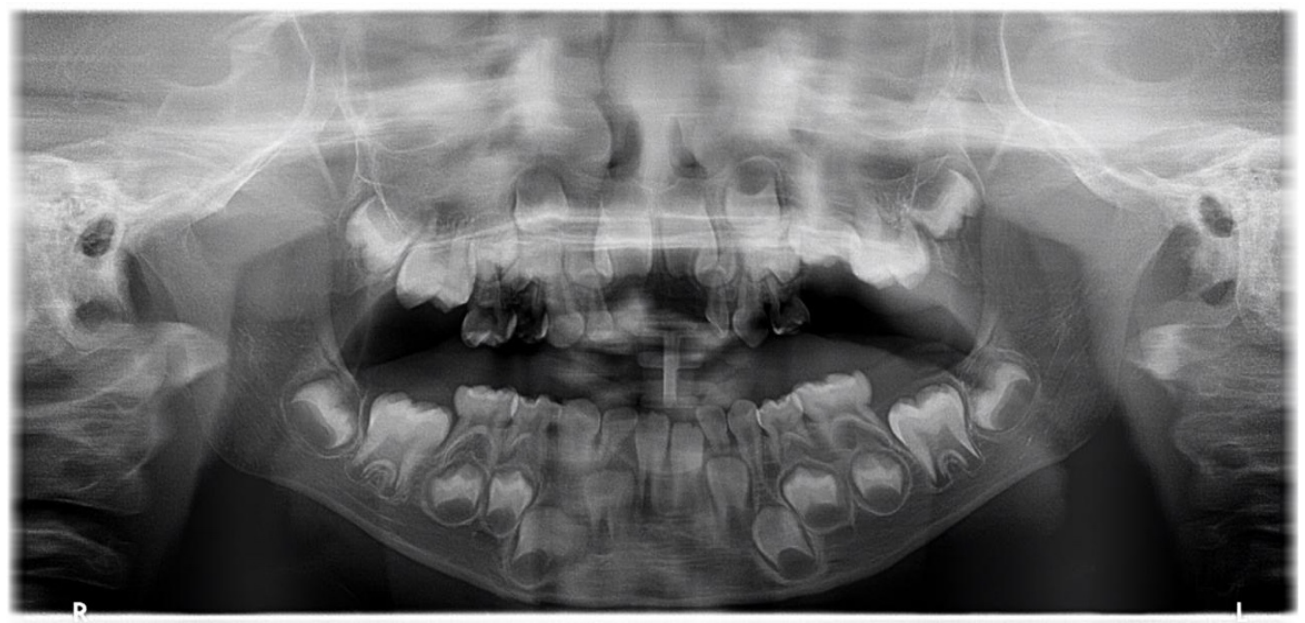

Fonte: Autores.

Figura 2: Imagens da evolução do processo de irrupção dos dentes 11 e 21. Fotos realizada pela mãe a cada telemonitoramento.

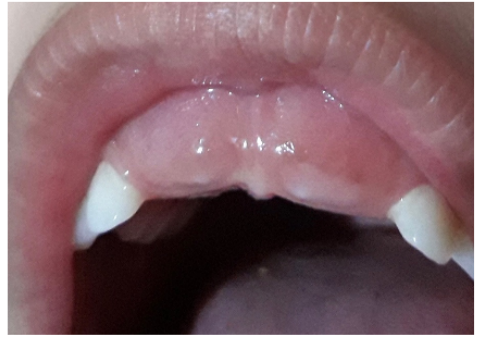

15 Novembro 2020

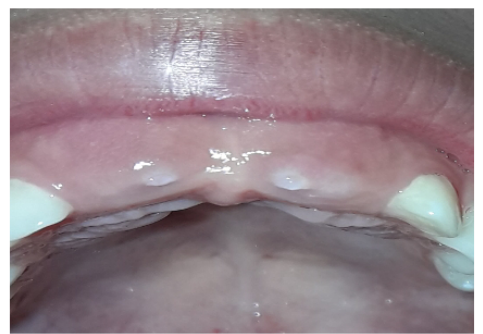

27 Novembro 2020

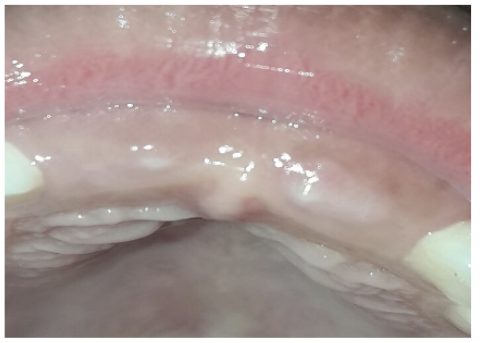

22 Novembro 2020

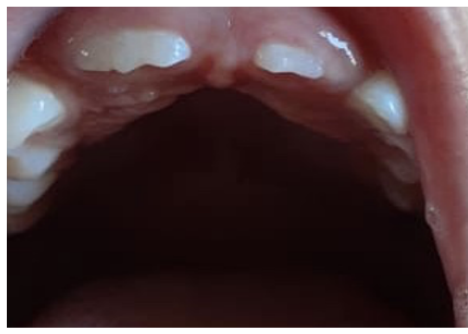

20 dezembro 2020

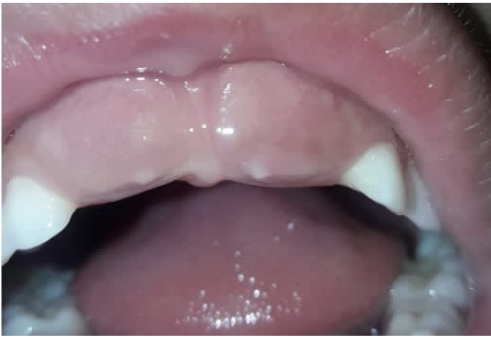

23 Novembro 2020

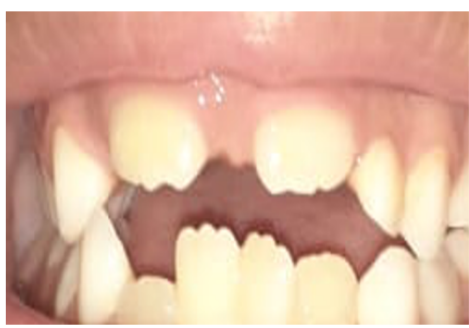

13 janeiro 2021

Fonte: Autores.

Figura 3- Registro final enviado pela mãe no dia 01 de fevereiro de 2021.

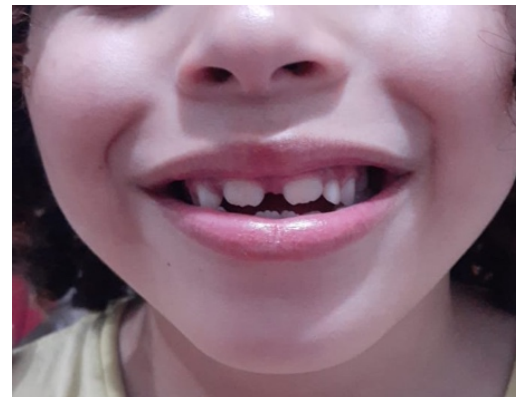

Fonte: Autores. 


\section{Discussão}

A Odontologia de Mínima Intervenção (OMI) é uma filosofia de cuidados que pode e deve ser aplicada a pacientes de qualquer idade e perfil socioeconômico. Nesta mudança de paradigma, as alternativas conservadoras são esgotadas antes da indicação de técnicas invasivas, complexas e mais onerosas para o paciente (Azar, 2020). Na clínica odontopediátrica, são comuns situações nas quais há a impaç̧ão de elementos dentários, fato que pode acarretar transtornos para a dentição em desenvolvimento. De acordo com a literatura, nessas situações pode-se pensar em alguns tratamentos para melhorar à função estética e com pós operatório favorável: exposição cirúrgica com tracionamento dentário, ulotomia, ulectomia (Cavalcanti \& Paiva, 2006). No caso apresentado, a ausência dos incisivos centrais estava causando ansiedade no paciente, 8 anos, uma vez que a demanda estética é importante para o desenvolvimento social, auto-estima e o confiança do paciente (Crawford, 1997; Suri et al., 2004). Um dado interessante reportado pela mãe, foi que seu filho, na época com 2 anos de idade, teve a perda prematura dos dentes decíduos (51 e 61) devido a avulsão dos mesmos em uma queda. Essa informação pode explicar a demora da erupção, uma vez que a perda precoce dos dentes decíduos pode deixar a gengiva local mais fibrosa (Feu, Rosetti Lessa, Awad Barcellos, Grillo, \& Freitas, 2018; Nadelman et al., 2020). A decisão do tratamento cirúrgico neste caso seria principalmente porque a criança teve uma perda dos incisivos centrais superiores decíduos há mais de seis meses e os incisivos centrais superiores permanentes não irromperam há mais de um ano após a irrupção dos incisivos inferiores permanentes. Porém, devido ao cenário pandêmico da COVID-19 e ausência de vacina para esse vírus, muitos países implementaram uma série de intervenções para reduzir a transmissão viral e frear a rápida evolução do contágio. Tais medidas incluíram o isolamento de casos; o incentivo à higienização das mãos, à adoção de etiqueta respiratória e ao uso de máscaras faciais; distanciamento social, com o fechamento de escolas e universidades, a proibição de aglomerações, a restrição de viagens e transportes públicos, a conscientização da população para que permaneça em casa, até a completa proibição da circulação nas ruas, exceto para a compra de alimentos e medicamentos (Credie et al., 2020; Kupferschmidt \& Cohen, 2020). Assim, a alternativa foi acompanhar o caso à distância usando o tele monitoramento. Para isso, toda semana era realizada uma chamada de vídeo com o responsável e a criança com objetivo de acompanhar a evolução do processo de irrupção, acolher e esclarecer sobre a situação. A teleconsulta tem sido reconhecida e regulamentada no Brasil como uma ferramenta auxiliar para ajudar nesse momento de pandemia de COVID-19 permitindo que os profissionais realizem a triagem, orientem, monitorarem visualmente pacientes com suspeita ou positividade para SARS-CoV-2 que apresentam lesões orais por meio de controle fotográfico e encaminhem os pacientes isoladamente para atendimento presencial, se necessário (Ghai, 2020; Odontologia, 2020; Revista de Odontopediatría Latinoamericana, 2021). Para que aja a erupção dentária, o dente realiza uma série de fases e movimentos intra osseos até passar pela via eruptiva rompendo o epitélio oral (Proffit \& Frazier-Bowers, 2009; Karla Mayra Rezende, Salgado, \& Bönecker, 2019). Nesse caso clinico o paciente apresentava um aumento da gengiva com coloração pálida, que se dá pela camada queratinizada do epitélio. No caso apresentado, o telemonitoriamento e aguardar a resolução espontânea clinica foi a conduta de acordo com a literatura que apresentam dados no qual indicam que a erupção espontânea do incisivo superior impactado pode levar até 3 anos (Mason, Azam, Holt, \& Rule, 2000; Mitchell \& Bennett, 1992; Smailiene, Sidlauskas, \& Bucinskiene, 2006).

\section{Considerações Finais}

O telemonitoramento ganhou um aumento exponencial no atendimento à distância durante a pandemia. Assim, no caso apresentado, antes de realizar algum procedimento cirúrgico foi feito o acompanhamento do paciente à distância que resultou em uma resolução clinica espontânea. Portanto, um diagnóstico criterioso e o conhecimento do caso é fundamental para uma correta da conduta podendo ser resolvido de forma simples e eficaz. 
Research, Society and Development, v. 10, n. 7, e18010716439, 2021

(CC BY 4.0) | ISSN 2525-3409 | DOI: http://dx.doi.org/10.33448/rsd-v10i7.16439

\section{Referências}

Azar, F. M. (2020). Minimally Invasive Surgery: Is Less More? Orthop Clin North Am, 51(3), xiii-xiv. doi:10.1016/j.ocl.2020.04.001

Cavalcanti, A. L., \& Paiva, L. C. A. (2006). Utilização da ulectomia na clínica infantil: relato de caso. UEPG Ci. Biol. Saúde, $12(3), 4$.

Couto, E. V. T. d., Franco, M. I., Bussadori, S. K., Santos, E. M., \& Rezende, K. M. (2021). Irrupção do primeiro molar permanente e o peso da criança. Há uma associação? Research, Society and Development, 10(3), 7. doi:10.33448/rsd-v10i3.13024.

Crawford, L. B. (1997). Impacted maxillary central incisor in mixed dentition treatment. Am J Orthod Dentofacial Orthop, 112(1), 1-7. doi:10.1016/s08895406(97)70266-X

Credie, G. B., Coelho, A. A., \& Rezende, K. M. (2020). Coronavirus (Covid-19) in Children: History and Pediatric Oral Health. Dental oral biology and craniofacial research 3(3), 4. doi:10.31487/j.DOBCR.2020.03.06

Dodson, T. B., \& Susarla, S. M. (2014). Impacted wisdom teeth. BMJ Clin Evid, 2014. Retrieved from https://www.ncbi.nlm.nih.gov/pubmed/25170946

Feu, D., Rosetti Lessa, F. C., Awad Barcellos, L., Grillo, C. B., \& Freitas, L. A. (2018). Factors Associated with Premature Loss of Primary Teeth in Brazilian Children. J Dent Child (Chic), 85(3), 108-113. https://www.ncbi.nlm.nih.gov/pubmed/30869586

Freitas, J. A., Carvalho, I. S., Alves, F. B., Costa, N. d. S., \& Carvalho, G. A. O. (2021). Biosafety in dental surgical procedures in front of the COVID-19 pandemic. Research, Society and Development, 10(1), 9. Retrieved from https://rsdjournal.org/index.php/rsd/article/view/11401.

Ghai, S. (2020). Teledentistry during COVID-19 pandemic. Diabetes Metab Syndr, 14(5), 933-935. doi:10.1016/j.dsx.2020.06.029

Golder, D. T., \& Brennan, K. A. (2000). Practicing dentistry in the age of telemedicine. J Am Dent Assoc, 131(6), 734-744. doi:10.14219/jada.archive.2000.0272

Haddad, A. E., \& Correa, M. S. (2005). The relationship between the number of erupted primary teeth and the child's height and weight: a cross-sectional study. $J$ Clin Pediatr Dent, 29(4), 357-362. doi:10.17796/jcpd.29.4.j10510371q155847

Jarvis, L., \& Stanberry, B. (2005). Teleradiology: threat or opportunity? Clin Radiol, 60(8), 840-845. doi:10.1016/j.crad.2005.04.001

Kupferschmidt, K., \& Cohen, J. (2020). Can China's COVID-19 strategy work elsewhere? Science, 367(6482), 1061-1062. doi:10.1126/science.367.6482.1061

Mason, C., Azam, N., Holt, R. D., \& Rule, D. C. (2000). A retrospective study of unerupted maxillary incisors associated with supernumerary teeth. Br J Oral Maxillofac Surg, 38(1), 62-65. doi:10.1054/bjom.1999.0210

Meng, L., Hua, F., \& Bian, Z. (2020). Coronavirus Disease 2019 (COVID-19): Emerging and Future Challenges for Dental and Oral Medicine. J Dent Res, 99(5), 481-487. doi:10.1177/0022034520914246

Mitchell, L., \& Bennett, T. G. (1992). Supernumerary teeth causing delayed eruption--a retrospective study. Br J Orthod, 19(1), 41-46. doi:10.1179/bjo.19.1.41

Nadelman, P., Bedran, N., Magno, M. B., Masterson, D., de Castro, A. C. R., \& Maia, L. C. (2020). Premature loss of primary anterior teeth and its consequences to primary dental arch and speech pattern: A systematic review and meta-analysis. Int J Paediatr Dent, 30(6), 687-712. doi:10.1111/ipd.12644

Odontologia, C. F. d. (2020). Resolução CFO-226. Retrieved from http://sistemas.cfo.org.br/visualizar/atos/RESOLU\%c3\%87\%c3\%83O/SEC/2020/226

Paules, C. I., Marston, H. D., \& Fauci, A. S. (2020). Coronavirus Infections-More Than Just the Common Cold. JAMA, 323(8), 707-708. doi:10.1001/jama.2020.0757

Proffit, W. R., \& Frazier-Bowers, S. A. (2009). Mechanism and control of tooth eruption: overview and clinical implications. Orthod Craniofac Res, 12(2), 5966. doi:10.1111/j.1601-6343.2009.01438.x

Revista de Odontopediatría Latinoamericana, E. d. t. m. d. 1. (2021). Teleodontologia: Aplicação à Odontopediatria durante a pandemia COVID-19. Journal of Latin American Pediatric Dentistry, 10(2), 20. doi:https://doi.org/10.47990/alop.v10i2.192

Rezende, K. M., Salgado, M. A. C., \& Bönecker, M. (2019). A odontogênese no entendimento da odontopediatria. In Napoleão (Ed.), Anuario de Odontopediatria- Integrada e Atual (Vol. 4, pp. 124). Campinas.

Rezende, K. M., Zollner, M. S., \& Santos, M. R. N. (2010). Avaliação da erupção dentária decídua em bebês considerados de risco. Pesquisa Brasileira Odontoped Clin Integr, 10(1), 5 .

Schneider, T., Filo, K., Kruse, A. L., Locher, M., Gratz, K. W., \& Lubbers, H. T. (2014). Variations in the anatomical positioning of impacted mandibular wisdom teeth and their practical implications. Swiss Dent J, 124(5), 520-538. Retrieved from https://www.ncbi.nlm.nih.gov/pubmed/24853188

Smailiene, D., Sidlauskas, A., \& Bucinskiene, J. (2006). Impaction of the central maxillary incisor associated with supernumerary teeth: initial position and spontaneous eruption timing. Stomatologija, 8(4), 103-107. https://www.ncbi.nlm.nih.gov/pubmed/17322650

Suri, L., Gagari, E., \& Vastardis, H. (2004). Delayed tooth eruption: pathogenesis, diagnosis, and treatment. A literature review. Am J Orthod Dentofacial Orthop, 126(4), 432-445. doi:10.1016/j.ajodo.2003.10.031 